\title{
Una breve introducción al derecho de los tratados
}

\author{
Antonio Alejandro Vega Bartra
}

A don Alfredo Eskenazi L. (M. D.), hombre sabio y bueno.

\section{Introducción}

El fin de este trabajo es responder una sola pregunta: ¿qué es un tratado? Debemos confesar que es una interrogante que nos hemos planteado en numerosas ocasiones, algo que probablemente también lo hagan muchos estudiantes y profesionales. He allí el origen de nuestro interés y el prurito que nos lleva a tratar de esbozar una respuesta.

No tenemos la intención de desarrollar aquí una investigación extensa, que exhiba todas las aristas de la negociación, formación, celebración y ratificación de los tratados, sino simplemente contrastar la práctica - algunos dirían, la "conducta" - de los Estados en el día a día de sus relaciones, con las costumbres (jurídicas) y normas existentes sobre los tratados.

La investigación combina, en la medida de lo posible, de forma clara, la evolución de la costumbre y prácticas que han constituido vínculos jurídicos, con un registro histórico, entre "soberanos" (entiéndase esto como un acuerdo o "documento" - en un sentido muy lato- que vincula a dos territorios, naciones, o en lo que en nuestro tiempo entendemos como dos o más Estados).

* El presente trabajo fue premiado en el Concurso de Actualidad Jurídica José León Barandiarán Hart. 
No hemos podido escapar a la necesidad de presentar un análisis histórico, pero, en beneficio del lector - al menos, eso esperamos-, solo hemos de presentar las prácticas de los pueblos más antiguos, en un esfuerzo por demostrar que muchas de las instituciones o costumbres (jurídicas) que creíamos "clásicas" o modernas, datan de un tiempo mucho más remoto.

Finalizamos el trabajo con el análisis de un caso que consideramos incluye muchas de las explicaciones hechas en el texto y que incorpora el razonamiento jurídico de la Corte Internacional de Justicia, confirmando los conceptos, costumbres o espíritu de las normas existentes de lo que se podría considerar el sistema internacional de ese entonces. No está de más decir que cualquier error $u$ omisión es totalmente atribuible al autor.

\section{Los tratados como instrumentos jurídicos internacionales}

En las relaciones entre Estados, la práctica hace evidente que existe una innegable necesidad de contar con instrumentos jurídicos suficientes para asumir obligaciones y cumplir compromisos internacionales, reconocer derechos, formar o incorporarse a organizaciones de naturaleza internacional o participar, como parte, en los fines de un bloque de integración. Estos instrumentos reciben el nombre genérico de tratados.

Por estos motivos, entre muchos otros, los tratados se convierten en el principal instrumento jurídico y una de las fuentes del derecho internacional más utilizadas por los Estados, especialmente cuando tienen la intención de imprimir un cariz de solemnidad, revestir de importancia un acuerdo entre (Estados) soberanos o tratar una materia particularmente sensible.

Es a partir de los intereses de estos mismos Estados, de vincularse dentro de la sociedad internacional, en hacer cumplir las obligaciones pactadas, hacer valer sus derechos o interpretar en su favor o en su defensa las cláusulas de un tratado, que el llamado derecho de los tratados cobra una importancia muy grande para la negociación de instrumentos jurídicos de carácter internacional y la interrelación que puedan tener entre Estados al mismo nivel, puesto que el tratado se convierte en el principal vehículo jurídico formal, diseñado para contener compromisos, obligaciones y/o derechos dentro del derecho internacional público.

Desde una perspectiva clásica - por no decir histórica -, los tratados sirven también como el testimonio, como una "memoria" de la 
práctica histórica de la sociedad internacional ${ }^{1}$, ya que mediante su constante uso se fomenta la progresiva evolución de la materia en particular (del derecho de los tratados) y la expansión del derecho internacional en su conjunto.

Y es que, por ejemplo, desde la Paz de Westfalia (1648) en adelante -época en la que el objeto de los tratados internacionales era generalmente la paz, la delimitación de territorios y fronteras, además de las alianzas políticas y militares-, estos instrumentos se han visto envueltos en un constante proceso de evolución en su alcance y contenido.

Es en el siglo XIX cuando se empiezan a celebrar un mayor número de tratados, con un contenido más técnico, abarcando - entre otrasmaterias administrativas o industriales. Se crearon con mayor presencia internacional alianzas económicas, las uniones aduaneras, regulando el comercio o refinando - con la evolución de la conciencia de la sociedad internacional - organizaciones de integración.

Durante el siglo XX, y más aún después de los trágicos eventos de la primera y la segunda guerras mundiales, se concluyen o se vuelve a resaltar la importancia de tratados orientados a la búsqueda de la solución pacífica de las controversias o, con diferente objeto, pero con un espíritu similar, tratados constitutivos de organizaciones internacionales de carácter mundial - como las Naciones Unidas-, fenómeno que "refleja la voluntad de responder a problemas y necesidades sociales compartidas mediante reglas jurídicas pactadas" (Remiro, 2007, p. 314).

\section{Inicios y breve reseña de su práctica histórica}

Es imposible relatar con rigurosidad académica toda la historia relevante del derecho internacional en una investigación que pretende ser breve. Por ello, el ejercicio metodológico nos obliga a sintetizar, en la medida de nuestras posibilidades, muchos años de prácticas entre territorios "soberanos" y sus costumbres "diplomáticas". Partiendo de esta afirmación, resultaría más difícil aún desarrollar con minuciosidad absoluta la historia del derecho de los tratados, por lo que, para una

1 “En un sentido amplio, una sociedad es internacional cuando el poder está descentralizado, distribuido entre grupos que lo monopolizan en sus respectivos territorios. [...]. Esencialmente, pues, la sociedad internacional es una sociedad de comunidades humanas con poder de autodeterminación, de entes colectivos autónomos" (Truyol y Serra, 2008, p. 20). 
comprensión razonable y apropiada del tema, presentamos, como solución y pidiendo la indulgencia del lector, distintos "hitos históricos" que exponen las principales características de esa historia a través de los años y correspondientes solo a un espacio de tiempo dentro de ella.

Abordaremos el tema en tres breves secciones: la primera tratará de la práctica del llamado "Mundo Antiguo", en especial, la práctica del Antiguo Oriente; la segunda explorará la fecunda práctica griega; y la tercera, en menor medida, la práctica romana.

Hay que confesar que en las reflexiones que acompañaban a nuestra investigación llegábamos a la conclusión de que lo mejor para el lector, y de forma consecuente con este trabajo, era que no nos correspondía tratar el periodo histórico clásico y moderno, es decir, el que comprende la evolución de la materia a partir del siglo XVII (Paz de Westphalia, 1648) en adelante.

Sería un esfuerzo titánico y, sobre todo, inoportuno, pues existen obras de mucho rigor académico y muy bien documentadas que desarrollan la historia y la evolución de lo que nosotros entendemos hoy por derecho internacional. Por ello, remitimos al investigador, profesional o estudiante que desee profundizar, a obras que se pueden considerar como referentes en el mundo de habla hispana ${ }^{2}$.

Nos detendremos únicamente en el punto que continúa a esta presentación histórica, a desarrollar con un acercamiento contextual la fuente material de lo que conocemos en nuestros días como "derecho de los tratados": la Convención de Viena sobre el Derecho de los Tratados de $1969^{3}$.

\section{a) El Mundo Antiguo}

Desde la más remota antigüedad, los príncipes y los Estados han celebrado tratados internacionales. En las vicisitudes de la guerra y de la paz que se entretejen en la trama de la historia, la semántica misma parece implicar que el establecimiento de la paz se encuentra vinculado a la celebración de pactos (Reuter, 1999, p. 13).

2 Por citar solo dos ejemplos, podemos recurrir a la obra del profesor Juan Carrillo Salcedo, El derecho internacional en perspectiva histórica, o a la del profesor Antonio Truyol y Serra, Historia del derecho internacional público, ambas publicadas por la editorial Tecnos.

3 Ver el apartado 4: La Convención de Viena sobre el Derecho de los Tratados. 
Para poder hablar de la historia que trata de la práctica jurídica que vinculaba a distintas unidades políticas organizadas, debemos partir tomando como dada una elemental presunción: en la antigüedad existieron civilizaciones que en su organización, conciencia colectiva e interrelación constituyeron - sin teóricamente serlo- lo que hoy denominamos Estados.

En esta sección tomaremos como principal texto de análisis el notable y profundo estudio del profesor David J. Bederman, International law in antiquity, que desarrolla con minuciosidad la práctica de interrelación jurídica entre pueblos antiguos, proponiendo como tesis principal de su estudio que en la práctica y en los hechos existió un primigenio derecho internacional.

La doctrina especialista en la materia identifica tres grandes periodos y ubicaciones geográficas que observaron una práctica similar a la que hoy consideramos como derecho internacional, y a un grupo de comunidades que conformaban una "sociedad de Estados" - en términos latos-. Los tres grupos a los que hace referencia son: a) el antiguo Cercano Oriente (1400 al 1150 a.C. y de 966 al 700 a.C.); b) las ciudades-estado griegas (500 a.C. al 338 a.C.); y c) las comunidades que florecieron bañadas por el Mediterráneo durante el periodo de expansión romana (358 a.C. al 168 a.C.) (Bederman, 2001, p. 16).

El contenido de estos primeros tratados no era uniforme en su fraseo y solemnidades, pero mantenían a lo largo y ancho de los territorios en los que se hacía uso de ellos una "unidad básica en su entendimiento", que se conservaba para los acuerdos "internacionales" celebrados por las culturas sumeria, egipcia, hitita, asiria e israelí ${ }^{4}$. Es decir, podían variar ligeramente las formas, pero se mantenía la esencia del instrumento.

Entre los tratados más antiguos y más importantes de los que se tiene registro encontramos, en primer lugar, el concluido por las ciudadesestado sumerias de Lagash y Umma alrededor del II o III milenio antes de Cristo, acuerdo que, grabado en una estela de piedra, con las fórmulas religiosas y místicas que rodeaban a los asuntos de Estado en ese periodo histórico, establecía solemnemente los límites entre sus territo-

4 "[...] some scholars have suggested that there is an essential unity in the the ancient oriental treaty pattern'" (Bederman, 2001, p. 138). Esto debe entenderse como una toma de conciencia de la función y esencia propias de estos tipos de instrumentos "jurídico-internacionales". 
rios, obligando a sus gobernantes a respetarlos. Los hombres de Umma debían jurar, por varios de sus dioses más poderosos, que observarían los términos del tratado y que su violación acarrearía su destrucción o, incluso, la muerte.

El hecho de que solo se tomara a un grupo determinado de dioses y de que juraran solo los hombres de Umma sugeriría un cierto estatus de vasallaje o de dependencia frente a Lagash. Por último, en las cláusulas que fueron negociadas entre las partes, la doctrina especula que se nombró a Mesilim de Kish, príncipe de una región vecina, como un garante de los límites fronterizos o como árbitro de cualquier controversia que pudiera originarse y que fuera materia del tratado ${ }^{5}$.

Existe cierta controversia acerca de la fecha exacta de este tratado, pues, por ejemplo, en su libro International Law, el notable jurista Vaughan Lowe precisa la fecha hacia el II milenio antes de Cristo, citando la investigación de un jurista del siglo XVIII:

Jean Barbeyrac, an eighteenth-century french jurist who translated several of the seminal early texts on international law, published in 1739 a collection of treaties reaching back into the second millenium BCE. There is, for example, a Sumerian treaty from around 2500 BCE on the Stele of Vultures, now Held in the Louvre, which records the establishment of the boundary between the kingdoms of Lagash and Umma (Lowe, 2007, p. 65).

Por otro lado, el internacionalista Arthur Nussbaum, en su clásica obra Historia del derecho internacional, ubica al mismo tratado en el III milenio antes de Cristo:

Hacia 3100 (a.C.), aproximadamente, se celebró un tratado entre Eannatum, el victorioso señor de la ciudad-estado de Lagash, en Mesopotamia, y los hombres de Umma, otra ciudad-estado de la misma región. El tratado se redactó en el idioma sumérico, y se ha conservado en una inscripción existente en un monumento de piedra (estela) que se descubrió en la primera década de este siglo [siglo XX] [énfasis nuestro] (citado por Novak \& García-Corrochano, 2003, p. 19).

5 "The men of Umma had repititively to swear by their gods that they would observe the terms of the peace treaty. If they did not, 'the great net'-the favored instrument of mayhem and destruction of at least one of the named gods'would destroy them'” (Bederman, 2001, p. 139). 
Las discusiones con respecto a la fecha exacta del tratado son importantes para el mundo académico, sin duda, pues nos proporcionan datos de la ubicación temporal en la que determinadas prácticas jurídicas se usaban entre distintas culturas. Además de la distinción histórica, lo que debemos rescatar es la solemnidad (que hoy llamaríamos "forma") en la celebración del tratado, y la vinculación, la "obligatoriedad" que los señores de cada territorio tomaban como asumida por estos como representantes de su "Estado" (es decir, un primigenio principio del pacta sunt servanda).

Otro tratado que por su antigüedad e importancia es preciso mencionar es el celebrado mediante un intercambio de $\operatorname{cartas}^{6}$ por Ramsés II, faraón de Egipto, y Hattusili III, rey de los hititas, en 1280 a.C., concertando la paz y la amistad entre sus reinos ${ }^{7}$. Este tratado es, tal vez, el único que puede ser analizado en los textos originales y en la forma en que fueron redactados por las partes, razón por la cual se le considera entre los más importantes de la antigüedad en haber sido descubiertos, pues se encontraron copias en el templo de Amón, en la capital del Egipto dinástico (Karnak), y en Ramesseum, ubicado en Asia Menor, en ese entonces territorio hitita (Bederman, 2001, p. 146).

En palabras del propio Bederman:

The 1280 BCE agreement between the Hittites and Egyptians stands as the epitome of parity treaties in the ancient Near East. The structure of the clauses, the phrasing of the language, and the relatively modest scope of the provisions indicate a desire for two great powers to regulate the most basic elements of their political relationship. As a paradigm of the ancient Near Eastern treaty, this instrument would have and enduring influence on later traditions of treaty-making in the ancient world. Its inspiration would first be felt in Israelite practice, and then in Greek and western Mediterranean treaty models [énfasis nuestro] (2001, p. 150).

6 “[...] resulta interesante saber que los tratados entre el faraón de Egipto y el rey de los hititas se concluyeron mediante un intercambio de cartas [...]" [o lo que hoy puede ser interpretado como un intercambio de cartas] (Reuter, 1999, p. 13).

7 En este sentido, detallan la práctica histórica de este tratado los internacionalistas Remiro Brotons (2007, p. 313) y Novak \& García-Corrochano (2003, pp. 19-20). 


\section{b) Práctica griega}

No hay fuente del saber en el mundo occidental, al día de hoy, que no haya sido influenciada de una u otra manera por la cultura y filosofía de la Antigua Grecia. El derecho, entonces, y en particular el derecho internacional, no pueden ser la excepción.

El mundo griego fue, durante ese periodo histórico (alrededor del 338 a.C.), una de las civilizaciones con una de las prácticas jurídicas y diplomáticas más sobresalientes, tanto por su complejidad en la precisión de los términos y el léxico que empleaban en sus embajadas a otras ciudades-estado, como por los diversos tipos de instrumentos que utilizaban, dependiendo de la naturaleza de la materia que intentaban "consagrar" (Bederman, 2001, p. 154).

Los tipos de tratados que se conocieron y se emplearon en la Antigua Grecia fueron variados, desde tratados de paz, alianza, amistad, asistencia, hasta tratados comerciales o tratados que tenían un marcado interés religioso o de culto, entre otros.

Se ha dicho, y no sin razón, que la vigencia de los tratados en el Mundo Antiguo era limitada. Una ciudad-estado podía celebrar un tratado de paz "a perpetuidad" con otra, sin que ello significara su eterna amistad o la prohibición o exclusión absoluta de un futuro conflicto diplomático o bélico.

In spite of these suggestions that the Greeks saw the treaty as an instrument to obtain universal peace and order, the evidence strongly contradicts that conclusion. To the extent that peace treaties were imposed upon a vanquished polity, they inevitably recognized the political hegemony and supremacy of the victor. When a peace treaty was made between two equal powers, in circumstances indicating that neither had defeated the other, then they were always for a limited period, usually for never more than fifty years (Bederman, 2001, p. 158).

Un tratado de philia (amistad) se distinguía de una symmachia (alianza) por su aparente mayor grado de "fuerza", de poder vinculante. Mientras que el primero se podía considerar como el instrumento jurídico más elemental (considerado por algunos como una simple formalidad) en cuanto a relaciones entre ciudades-Estado, o el primer paso para profundizar o fortalecer las relaciones entre estas, la última entrañaba un mayor grado de complejidad y obligaciones expresas. Los 
argumentos que se esgrimen a favor de una relación de tácita jerarquía entre uno y otra llevan a considerar a esta última como un instrumento jurídico más complejo y solemne: "In short, two polities could be friends, and yet not be allies. [...] Neutrality, by virtue of contrary sets of philiai, came whith a price. A friend to all was a friend to none" (Bederman, 2001, pp. 159-161) (énfasis nuestro). Una distinción adicional se hacía entre alianzas de naturaleza militar: con la celebración de una symmachia se concluía una alianza ofensiva, diferente de la epimachia, que era de carácter meramente defensivo. Mientras que dos ciudades-estado se debían asistencia mutua en caso de un conflicto bélico - symmachia de por medio-, la epimachia solo obligaba a brindar asistencia a la otra en caso de que esta sufriera una invasión ${ }^{8}$. En contraste, las formas más complejas y desarrolladas de instrumentos jurídicos internacionales - en ese periodo histórico - , para la práctica griega, eran lo que se podría considerar como la unión federal de ciudades-estado (sympoliteiai), o las ligas internacionales $\mathrm{u}$ organizaciones de ciudades-estado que tenían por centro o carácter común un elemento religioso o de culto (amphictyoneiai).

Un ejemplo digno de explicar, por la habilidad del razonamiento jurídico de los "plenipotenciarios" y el principio del derecho que involucra dentro de la práctica griega, es el sucedido entre las ciudadesestado de Acarnania, Esparta y Aetolia.

¿Existían los conflictos entre "tratados" en la antigüedad? Por lo que relata Polibio en sus Historias ${ }^{9}$, un aparente conflicto de obligaciones (entre tratados) surgió cuando el enviado de Acarnania solicitó a los espartanos su ayuda en una guerra contra Aetolia, invocando una symmachia entre estos, sin contar con que los aetolios habían celebrado una epimachia con Esparta.

La solución dada resulta sorprendente, por la habilidad retórica del orador y la relevancia histórica que entraña, pues haciendo una pregunta abierta, dejó a la consideración y posterior decisión de los espartanos el elegir qué obligación era la más sagrada de honrar: un acuerdo entre privados (en este caso, la epimachia) o un tratado que había sido inscrito en una columna de piedra y consagrado ante toda Grecia.

8 "In short, symmachiai were offensive alliances while epimachiai were defensive in nature. This distinction seems to have developed a full articulation only in the fifth century BCE" (Bederman, 2001, p. 162).

9 Autor en el que se apoya Bederman para construir su argumento. 
The first premise of Lyciscus' submission to the Spartans was surely correct: in ancient Greek practice a general treaty prevailed over a "private" arrangement. General peaces were held to cancel all outstanding networks of symachiai" (Bederman, 2001).

Y por si el primer argumento no conseguía la decisión deseada, añadió a su demanda de ayuda una alternativa "temporal o circunstancial" a la interpretación del aparente conflicto entre los dos "tratados": si las circunstancias en las que se firmó el tratado con los aetolios seguían siendo las mismas, sus obligaciones para con ellos permanecían intactas. Pero, de haber cambiado y encontrarse en un escenario completamente nuevo, no existía obstáculo "legal" alguno para deliberar y someter a voto la ayuda solicitada.

This is rightfully regarded as the first invocation of the doctrine of rebus sic stantibus in international law - the principle that one party may unilaterally terminate a treaty because of an intervening, fundamental change of circumstances [énfasis nuestro] (Bederman, 2001).

Después de haber explicado la práctica helena, no sería apresurado o inexacto afirmar que las instituciones jurídicas y los usos que pensamos "clásicos" (o de factura romana), en muchos casos no lo son, pues representan, al día de hoy, las prácticas o las costumbres de pueblos antiguos, que fueron ensombrecidas por el paso del tiempo y mantenidas o recordadas solo por un puñado de funcionarios o muy pocos estudiosos y, en otros casos, renombradas o "redescubiertas".

\section{c) Práctica romana}

The comparatively infrequent treaties of the Romans were for the most part concluded under the Republic, and they are on the whole not good examples of international law. Most of them reflect in terse technical phraseology the Roman methods of political expansion.

Arthur Nussbaum

En el tiempo de la antigua Roma, las relaciones entre distintas urbes y civilizaciones no eran ajenas a la práctica de sus gobernantes. Los romanos hicieron uso, principalmente, de dos tipos de tratados para relacionarse "internacionalmente" con los reinos o comunidades que entraban en su esfera de influencia o interés geográfico: la deditio y el foedus iniquum. 
La consolidación de su política exterior a través del mar - no olvidemos la frase: mare nostrum -, que Roma desplegó entre los años 250 a.C. al 50 a.C., la obligó a desarrollar instrumentos jurídicos que pudieran servir como herramientas para consolidar su hegemonía y sus campañas de expansión y "majestad" (maiestas) - la que se entendía en una relación de subordinación o vasallaje-, sobre los pueblos que conquistaban o con los que celebraban alianzas en un evidente estado de desigualdad.

Un modus vivendi - en ese contexto- que estaba caracterizado por las constantes campañas bélicas (o diplomáticas) de expansión y conquista, debía contar con instrumentos jurídicos que legitimaran su hegemonía. La deditio, entonces, se caracterizaba por ser la aceptación que consumaba la pérdida de la batalla o demostraba - sin haber entrado en ellaque las fuerzas romanas (militares y, luego, en consecuencia, políticas) eran superiores al reino o pueblo con el que se celebraba. Con este documento que, previo juramento ${ }^{10}$, ponía a disposición romana todas sus posesiones y personas (lo que hoy llamaríamos ciudadanos), se confirmaba la rendición o sumisión incondicional a Roma.

Sin embargo, la práctica política romana permitía que, en muchos casos, los pueblos y las comunidades vencidas conservaran cierto grado de autonomía y privilegios en su gobierno interior, así como la supervivencia de sus leyes y costumbres ${ }^{11}$.

Para las relaciones más complejas, con comunidades con las que se deseaba celebrar un tratado de alianza, el tratado que era de uso frecuente se denominaba foedus iniquum. Esta "alianza desigual", que ponía en evidencia la superioridad o la supremacía de Roma para con su "aliado" (¿Estado vasallo/cliente?), no era más que una expresión de su política exterior.

The Romans thus created a significant distinction between various forms of subordinate allies. A client State could be an amicus (friend) or a foederatus (ally), and the ruler of such states would be called by the Romans a king (rex) or phylarch or ethnarch. In the late nineteenth cen-

10 "[..] urbem, agros, aquam, terminos, delubra, utensilia, divina humanaque omnia". Citado por Bederman (2001, p. 189, nota al pie 336).

11 "A deditio on these terms fully subjugated the conquered nation to Roman will, although the Romans were known to have granted rights and privileges to peoples so vanquished, including a measure of autonomy and the right to continue the observance of their laws and customs" (Bederman, 2001, p. 189). 
tury, Mommsen developed a scheme distinguishing between different Roman client State relations. He identified three groups of states: (1) amici, who had no more than friendly relations with Rome; (2) amici et socii, those who in addition to friendly relations had a duty send military contingents to Rome upon request; and (3) socii, who were virtually subordinated to Rome and were required to provide set military contingents on an annual basis. Other scholars, including Bruno Paradisi, have speculated that the status of foedus with Rome was quite distinct from that of an amictia. The amicus relationship was premised on a renunciation of war; it was (in effect) the western equivalent of the Greek philia [énfasis nuestro] (Bederman, 2001, p. 190).

La práctica histórica del llamado mundo antiguo, como se explicó al inicio de esta sección, es difícil de sintetizar en una investigación de esta naturaleza. Baste decir que, para las civilizaciones que nacieron y se desarrollaron antes del nacimiento de Cristo, hubo una marcada similitud en las características que rodearon al entendimiento del concepto "tratado"12.

Hemos llegado a un punto en que se presentan dos caminos que son inseparables. Uno nos invita a seguir y profundizar, aún más, en el análisis de las instituciones jurídicas desde una perspectiva histórica, y otro nos urge a explorar el ingente cuerpo normativo que nace con la formación de los estados-nación y el advenimiento de la "soberanía", tal y como hoy la entendemos. Por las razones que hemos expuesto al inicio de este análisis, no nos adentraremos en esos temas, por lo que el desarrollo de esta materia queda, felizmente, pendiente para otro trabajo. Nos permitimos provocar la curiosidad del lector con una cita que esperamos sea el punto de partida para sus propias investigaciones:

12 "Anciente treaty-making also had a strong universalist flavor. By this I mean two distinct things. The first is that there was a single ancient tradition in treaty-making, an inherent unity of conception in the way that treaties were made and observed. There were, of course, some variations in these forms, but what is surprising is the commonality. The basic Hittite treaty form, a secular contract formed out of solemnized oaths, was adopted (with some changes) by the Assyrians, Egyptians, and ancient Israelites. It was, in turn, transmitted into the Greek and Hellenistic worlds, and thence into the western Mediterranean region of Rome and her neighbors. [...] it may have been the dropping of elaborate preambular passages, or the inclusion of specific legal terms or provisions (such as maisteas clauses or qualifications regarding after-acquired allies). Regardless, there was always a synthesis of forms and motifs of legal expression in treaties, never an outright repudiation of old forms" [énfasis nuestro] (Bederman, 2001, p. 205). 
El derecho público europeo (droit public de l'Europe, jus publicum Europaeum), también llamado - especialmente por los publicistas germanos - "derecho de gentes europeo" (europäisches Völkerrecht), ha sido la base del derecho internacional "clásico", que ha permanecido en vigor prácticamente hasta la primera guerra mundial. Sus cimientos fueron puestos por la Paz de Westfalia (1648), [...] punto de partida de toda una serie de tratados posteriores que a ellos se refieren y se enlazan expresamente unos con otros, formando un verdadero corpus iuris gentium europeo (Truyol y Serra, 2008, p. 36).

\section{La Convención de Viena sobre el Derecho de los Tratados}

Corría el año 1969 cuando, después de casi dos décadas de debates - pues se iniciaron en 1949- y de múltiples conferencias internacionales, se adoptó el texto final de lo que se conoce hoy como la Convención de Viena sobre el Derecho de los Tratados ${ }^{13}$.

La práctica jurídica con respecto al régimen aplicable a los tratados tiene mucho más años que los transcurridos desde la adopción y posterior apertura a ratificación de la Convención, por lo que no es exagerado afirmar que, con los trabajos de codificación de la Comisión de Derecho Internacional, "el derecho de los tratados ha tomado un nuevo punto de partida" (Reuter, 1999, p. 28), el que ha servido para ordenar y, en muchos casos, desarrollar la práctica de los Estados en la materia hasta ese entonces.

Gran parte de los artículos de la Convención trata sobre reglas de derecho existentes que versan sobre tratados; es decir, se la considera en esa gran parte como declarativa, "codificadora" de derecho internacional consuetudinario. Otras secciones y artículos de la Convención, sin embargo, incorporan un desarrollo y reglas que van más allá de la práctica establecida hasta la fecha de su adopción -entiéndase, hasta 1969-, o alteraron en todo o en parte reglas que se tomaban como establecidas ${ }^{14}$, por lo que se la considera, en términos generales, como la codificación de la costumbre internacional, formada por la práctica de los Estados respecto a los tratados, pero con precisiones que permiten fortalecer esa misma práctica o dar solución a interrogantes a los que la costumbre no había podido dar respuesta de manera eficaz o llegar a ser aceptada por los Estados en su mayoría.

13 United Nations Treaty Series 331, 8 International Legal Materials 679 (1969).

14 Damrosch et al. (2009, p. 123). Cfr. Brownlie (2008, p. 608). 
Durante el primer periodo de sesiones de la Comisión de Derecho Internacional se determinó que el derecho que regulaba los tratados era susceptible de codificación y, por lo tanto, materia de estudio. Fue así que a partir de 1949 en adelante, y por casi veinte años, se llevaron a cabo largos periodos de reflexión y discusión académica, lo que dio como fruto diversos informes que sirvieron a la comisión para presentar un borrador de proyecto final a la Asamblea General de las Naciones Unidas en 1966, proyecto que fue aceptado como principal documento a ser debatido en el primer periodo de sesiones de la Conferencia de Viena de 1968, y en el segundo, celebrado en $1969^{15}$.

Entre los notables y eminentes juristas británicos que se sucedieron en la tarea de dirección de tan grande esfuerzo se encuentran James Brierly, sir Hersch Lauterpach, sir Gerald Fitzmaurice y sir Humphrey Waldock. Los tres últimos fueron elegidos en su tiempo, sucesivamente, por sus méritos y conocimientos, jueces de la Corte Internacional de Justicia (Damrosch et al, 2009, p. 123).

La importancia que cobra la Convención de Viena sobre el Derecho de los Tratados es tal, que es reconocida como uno de los esfuerzos de debate y codificación más importantes en la historia del derecho internacional.

De la lectura de los artículos más relevantes para el propósito de este trabajo, debemos empezar por hacer notar que la Convención solo se aplica a los tratados concluidos entre Estados (artículo 1) y a los que hayan sido celebrados después de su entrada en vigor -27 de enero de 1980 - (artículos 4 y 84). Es decir, respetando el principio de irretroactividad, se aplicará solo a los tratados que sean celebrados, con posterioridad a la fecha de la entrada en vigor de la Convención, por los Estados que forman parte de ella (con una excepción que se señalará más adelante). Cabe agregar que el hecho de no haber ratificado la Convención no impide invocarla ante un tribunal internacional o en una controversia internacional, como un esfuerzo concreto de sistematización de la costumbre internacional.

Una de las locuciones latinas más repetidas en derecho internacional - reconocida como principio general del derecho por la práctica y la doctrina- es la de pacta sunt servanda. No solo contiene la obligación de los Estados de honrar sus compromisos, cumplir sus acuerdos, sino el deber

15 Resolución 2166 (XXI) del 5 de diciembre de 1966, Asamblea General de las Naciones Unidas. 
de llevarlos de buena fe en su negociación, celebración y cumplimiento, sin la posibilidad y validez de argumentar un conflicto de normas con su derecho interno como motivo de incumplimiento (artículos 26 y 27).

Especial atención merece la formulación del principio de irretroactividad para con las disposiciones de un tratado (artículo 28), pues si bien respeta la regla general al no aplicarse a los hechos o situaciones sucedidas con anterioridad a la entrada en vigor de sus cláusulas, deja abierta la posibilidad para excepciones: “... salvo que una intención diferente se desprenda del tratado o conste de otro modo", como puede leerse al final del artículo que analizamos.

Las excepciones al principio de irretroactividad son las siguientes:

a) Cuando las partes en el tratado así lo hayan convenido - como en el párrafo $1 .^{\circ}$ del anexo a la sección $V$ del Tratado de Versalles, por el que se reintegró a la nacionalidad francesa a los habitantes de Alsacia y Lorena con efectos del 18 de noviembre de 1918, fecha en que se recuperan los territorios, aunque el Tratado de Versalles se firmó el 28 de junio de 1919 y entró en vigor varios meses después.

b) Cuando la retroactividad se deduzca del propio tratado - por ejemplo, los acuerdos adicionales o acuerdos interpretativos de una convención, cuyos efectos hay que remontarlos al momento de la convención llamada principal o básica o del acuerdo que se interprete - o conste de otro modo (Diez de Velasco, 2007, p. 172).

Se ha hecho énfasis, y de forma expresa, que existen algunos temas que no pueden ser sujetos a las reglas generales prescritas por la Convención: casos como el de la ruptura de hostilidades entre Estados, el de una controversia o pretensión que tenga intención de atribuir responsabilidad internacional a un Estado, o el de sucesión de los mismos (artículo 73). No hay que olvidar que en uno de los casos en concreto existe un proyecto de artículos sobre responsabilidad internacional de los Estados por hechos internacionalmente ilícitos, aprobados por la Comisión de Derecho Internacional en 2001, que sirve como referente de la costumbre internacional relativa a la responsabilidad de Estados, aun así no haya sido ratificada.

\section{El tratado entendido en el análisis de sus características}

Ya sea que se le denomine acuerdo, pacto, convención, intercambio de notas o se le atribuya cualquier título similar, el académico, el operador jurídico y el juez deberán tener en cuenta que siempre será considerado 
como tratado - desde una perspectiva jurídica - el instrumento (jurídico) del cual se desprendan ciertas características que lo configuren como tal, entiéndase esto como el conjunto de características que a la luz del derecho internacional lo configuren como un "tratado".

Cabe recordar que la Convención de Viena sobre Derecho de los Tratados - el "Tratado sobre los Tratados" - , en su artículo segundo (1.a) utiliza un lenguaje muy amplio, que reúne en su redacción un conjunto de "características" que permiten el análisis, desde diferentes puntos, de un documento en particular para que pueda ser calificado o no como un tratado en términos jurídicos.

(Artículo 2.- Términos empleados)

1. Para los efectos de la presente Convención:

a) Se entiende por "tratado" un acuerdo internacional celebrado por escrito entre Estados y regido por el derecho internacional, ya conste en un instrumento único o en dos o más instrumentos conexos y cualquiera que sea su denominación particular; [...]

El artículo contiene en su redacción algunas características mínimas para poder calificar a un instrumento, documento o práctica internacional como un tratado. Es decir, el artículo, en su redacción y su fraseo, debe ser interpretado como un sistema que guarda estrecha conexión entre sus partes y los conceptos que puede contener.

Como una primera característica objeto de análisis, tomamos la frase: "... acuerdo internacional..."; esto es, un documento que tenga su origen o génesis en la práctica jurídica internacional entre Estados. Es pertinente hacer notar que la Convención no hace distinción alguna entre el tipo de tratado al que se le aplican sus relaciones jurídicas. De esta manera, se aplica tanto a los tratados bilaterales como a los multilaterales y, de más reciente data, a los tratados de integración económica.

No está de más señalar que, en el último supuesto, la Corte Europea de Justicia se ha encargado de aclarar, a través de su producción jurisprudencial, la pertinencia de la aplicación de las reglas de la Convención de Viena, en materia de interpretación - entre otros supuestos-, a sus tratados fundacionales ${ }^{16}$.

La segunda característica que se debe tomar en cuenta - con base en la redacción del artículo- prescribe un determinado "soporte" para que se considere un instrumento internacional como un tratado. 
Debe estar "... celebrado por escrito...", esto es, contenido en algún medio legible que permita su autenticación, por lo que no sorprendería poder considerar como "tratado" al instrumento internacional que se encuentre reproducido o escrito en un telegrama, un mensaje de fax $\mathrm{o}$, incluso, un e-mail.

Se considerarían, en estos casos, como documentos permanentes y legibles que pueden ser tomados como unos que cumplan el requisito de encontrarse en forma escrita, valga la aclaración, siempre y cuando las demás características concurran en el caso concreto (Aust, 2007, p. 19).

La celebración, en el contenido del artículo 2, obliga a que sea "... entre Estados...", lo que nos recuerda el alcance de la Convención, que solo pretende regular o tener efectos jurídicos sobre los instrumentos internacionales celebrados entre esas unidades políticas organizadas que llamamos Estados, a través de las ramas u órganos competentes o de sus representantes ${ }^{17}$.

Una cuarta característica es la que prescribe que un tratado, para ser considerado como tal, debe necesariamente encontrarse "... regido por el derecho internacional...". Por lo tanto, deberá tener en cuenta el elemento de la "intención" de crear obligaciones reguladas por el derecho internacional, pues, de no existir tal, el instrumento carecería de núcleo jurídico y no podría ser considerado como un tratado ${ }^{18}$. La Corte Internacional de Justicia se ha pronunciado al respecto y sobre este requisito ha declarado que debe existir una intención para concluir un tratado, la cual debe ser extraída de los términos y de las circunstancias en los que fue concluido el instrumento bajo análisis y no de lo que las partes puedan decir luego acerca de las que pudieron haber sido sus intenciones ${ }^{19}$.

16 Como, por ejemplo, en los casos C-312/91 Metalsa, T-115/94 Opel Austria, C-162/96 Racke.

17 Existe también, para las demás relaciones jurídicas con carácter internacional en las que puedan verse envueltos los Estados y como complemento de la Convención de 1969, la Convención de Viena sobre el Derecho de los Tratados entre Estados y Organizaciones Internacionales de 1986, que todavía no ha entrado en vigor.

18 "According to the International Law Commission's Commentary, the phrase 'governed by international law' embraces the element of an intention to create obligations under international law" [énfasis nuestro] (Aust, 2007, p. 20).

19 Aegean Sea Continental Shelf, Judgement, I.C.J. Reports 1978, p. 39, para. 96: [La Corte] "[...] it knows of no rule of international law which might preclude a joint 
La frase "... ya conste en un instrumento único o en dos o más instrumentos conexos..." reconoce que la categoría jurídica tratado ha estado, por mucho tiempo, en la práctica, acompañada por documentos celebrados y concluidos de forma menos convencional, pero que aun así mantenían dicha categoría: por ejemplo, un intercambio de cartas entre Estados, como sucediera en el caso Qatar v. Bahrain (1994), en el que la Corte tuvo que determinar el estatus jurídico de un doble intercambio de cartas y su respectiva relevancia para el caso que se estaba analizando. En este caso, ese mismo intercambio de cartas fue invocado como un tratado ante la Corte Internacional de Justicia y, a pesar de no haber sido inscrito/registrado como un tratado (art. 102.2 de la Carta de las Naciones Unidas), fue considerado como tal en todos sus términos por la Corte ${ }^{20}$.

La característica que puede presentarse como más confusa o controvertida, y cuyo significado no resulta obvio para el lego en derecho, dentro de la formulación del artículo 2, es la siguiente: “... y cualquiera que sea su denominación particular...". Y es que de su lectura y análisis se entiende que la frase final del párrafo incluye una "cláusula abierta" para la determinación del instrumento jurídico bajo examen, de manera independiente de su título. Con este enunciado, podemos afirmar sin reparos que el componente que resulta decisivo para su calificación como tratado es el llegar a determinar si los Estados que lo concluyeron tenían la intención de hacerlo o no hacerlo vinculante a la luz del derecho internacional (Aust, 2007, p. 23). Entonces, al analizar un instrumento jurídico que involucre como sujetos a dos o más Estados se tendrá

communiqué from constituting an international agreement to submit a dispute to arbitration or judicial settlement [...] the Court must have regard above all to its actual terms and to the particular circumstances in which it was drawn up".

20 "In Qatar v. Bahrain, the 1987 parallel Exchange of Notes that the parties agreed constituted a treaty were invoked before the Court, which gave full regard to their terms even though they had not been registered" (Aust, 2007, p. 104). Cfr. Clapham $(2012,307)$ : "In another dispute the Court had to determine the nature of, first, an exchange of letters, and second, Minutes of a meeting between the Foreign Minister of Qatar and Bahrain in the presence of the Foreign Minister of Saudi Arabia. The parties agreed, and the Court concluded, that the exchange of Notes constituted a binding international agreement. And the Court found that the Minutes of the subsequent meeting: 'enumerate the commitments to which the Parties have consented. They thus create rights and obligations in international law for the parties". Maritime Delimitation and Territorial Questions between Qatar and Bahrain, Jurisdiction and Admissibility, Judgement, I.C.J. Reports 1994, p. 112. 
que considerar, en primer lugar, siempre, su contenido, intención y fin, antes que su denominación.

Una vez determinado el tratado, debe considerarse, junto a la costumbre internacional y los actos unilaterales (en menor medida), como la principal fuente de obligaciones en el derecho internacional público.

\section{El incumplimiento de obligaciones como causa de responsabilidad internacional}

Se debe tener muy presente que existe una gran diferencia entre definir una norma y el contenido de la obligación que esta impone y, por otro lado, el determinar si esa obligación ha sido incumplida o constituye un acto ilícito y las consecuencias jurídicas que ello pueda acarrear.

Roberto $\mathrm{Ago}^{21}$

En 2001, la Comisión de Derecho Internacional aprobó, después de varias décadas de debates, un proyecto de artículos sobre responsabilidad internacional de los Estados por hechos internacionalmente ilícitos (Arsiwa, por sus siglas en inglés) ${ }^{22}$. Este documento - que aún no ha entrado en vigor - sistematiza la costumbre internacional en materia de responsabilidad atribuible a los Estados y que estos tomaban por aplicable. El

21 "The Commission agreed on the need to concentrate its study on the determination of the principles which govern the responsibility of States for internationally wrongful acts, maintaining a strict distinction between this task and the task of defining the rules that place obligations on States, the violation of which may generate responsibility. Consideration of the various kinds of obligations placed on States in international law and, in particular, a grading of such obligations according to their importance to the international community, may have to be treated as a necessary element in assessing the gravity of an internationally wrongful act and as a criterion for determining the consequences it should have. But this must not obscure the essential fact that it is one thing to define a rule and the content of the obligation it imposes, and another to determine whether that obligation has been violated and what should be the consequences of the violation. Only the second aspect of the matter comes within the sphere of responsibility proper; to encourage any confusion on this point would be to raise an obstacle which might once again frustrate the hope of a successful codification of the topic" [énfasis nuestro]. Ago, Roberto; ILC Yearbook, 1970, vol. II, p. 306, para. 66 (c).

22 Los artículos que se analizarán en este punto hacen referencia al proyecto en mención. 
proyecto desarrolla, considerablemente, un juego de reglas generales (lex generalis) a las que se ha de recurrir en caso de violación de una obligación internacional o de daño causado a un Estado y que tenga su génesis en la conducta u omisión de conducta de otro Estado. La responsabilidad internacional, así entendida, es producto, entonces, de la continua práctica de los Estados y de la jurisprudencia internacional en la materia; desde las primeras sentencias de la Corte Permanente de Justicia Internacional, hasta las de la contemporánea Corte Internacional de Justicia ${ }^{23}$.

El punto de partida para analizar un posible caso de incumplimiento de obligaciones internacionales es considerar, primero, la relación de responsabilidad que se crea a partir de estas reglas generales y que prescriben las consecuencias que le son atribuibles al Estado infractor por el hecho internacionalmente ilícito - o el daño, de haberlo-, lo que se denomina en doctrina reglas secundarias.

En contraste, y tomando como referencia la lúcida distinción del profesor Ago, las reglas primarias son las que establecen conductas $\mathrm{u}$ obligaciones exigibles a los sujetos de derecho internacional -en el caso, y para los efectos de esta investigación en particular: los Estados-, por lo que una violación de estas da inicio, debido a la dinámica que se ha sistematizado en el proyecto, a la determinación de las reglas secundarias aplicables y las consecuencias jurídicas que estas prescriban.

La responsabilidad internacional se relaciona con el incumplimiento de las obligaciones, y sus principios generales son los mismos, cualquiera que sea la fuente de la obligación (tratado, costumbre o acto unilateral) (Reuter, 1999, p. 223).

Se considera un principio general del derecho internacional el que un Estado asuma la responsabilidad de, o la carga por, las consecuencias jurídicas producidas a partir de un hecho internacionalmente ilícito atribuible a este (artículo 1). Para que se configure, entonces, esta acción u omisión ilícita que tiene origen en su "comportamiento", debe ser atribuible a ese Estado según las normas de derecho internacional y constituir la violación de una de sus obligaciones internacionales (artículo 2), aun en

23 La Corte Permanente de Justicia Internacional (CPJI) se estableció en virtud del Acuerdo de la Liga (Sociedad) de Naciones (1920). Fue predecesora, hasta 1946, de la Corte Internacional de Justicia (CIJ) como principal órgano judicial de las Naciones Unidas, la que fue creada por la Carta de las Naciones Unidas (1945) e inició sus trabajos en abril de 1946. 
el caso de que ese mismo hecho sea considerado lícito - legal- por el derecho interno del Estado cuestionado (artículo 3).

Un Estado no solo es responsable por los actos o comportamientos que deriven de él; son considerados, de igual manera, como factores atribuibles de responsabilidad, los actos o comportamientos que reconozca o adopte como propios (artículo 11) (este supuesto lo desarrollaremos con más detalle en un ejemplo posterior a este punto).

De manera adicional al análisis de los artículos, y a partir de una lectura sistemática del proyecto, se debe determinar si en el momento del origen de la violación de sus compromisos internacionales le era exigible al Estado una determinada obligación o conducta que se convierte en la causa misma de ese hecho ilícito (artículo 12).

Un principio muy importante para poder ubicar el ilícito en el tiempo y así determinar la norma aplicable a este es la llamada "regla intertemporal" 24 (artículo 13). Es decir, se debe tomar la norma vigente en el tiempo al momento de producido el hecho ilícito, sin importar, por ejemplo, si la controversia jurídica se presenta muchos años después. $Y$ se debe tomar, incluso, el mismo principio si la obligación desapareciera o ya no fuera exigible al Estado; es decir, cualquier responsabilidad surgida bajo ella sería tratada en relación con sus supuestos de hecho.

Los artículos del proyecto deben ser entendidos como un conjunto sistematizado de normas con un origen consuetudinario, por lo que de presentarse el caso de que una materia o supuesto no se encuentre "codificado" - utilizando la palabra en un sentido muy lato-, se deberá recurrir a la costumbre internacional (artículo 56). No hay que olvidar que los artículos son de aplicación general (¿residual/supletoria?); es decir, que cuando existan regímenes especiales de responsabilidad contenidos en tratados o en otras fuentes de derecho internacional, se ha de dar primacía a estas normas - por principio de lex specialis - (artículo 55).

Por último, el artículo 59 prescribe: “Los presentes artículos se entenderán sin perjuicio de la Carta de las Naciones Unidas", lo que debe

24 "This is a general principle of international law: an act must be judged according to the applicable international law at the time, not the law when a dispute about it arises, which could be many years later. The corollary is that, even if the obligation were to cease (for example, by termination of a treaty), any responsibility that had already accrued would be unaffected. The principle applies equally to a new jus cogens (peremptory norm)" [énfasis nuestro] (Aust, 2007, p. 382). 
interpretarse - en nuestra opinión - como una relación de tácita jerarquía en caso de conflicto entre la Carta y el proyecto.

\section{Personal diplomático y consular de Estados Unidos en Teherán (Estados Unidos c. Irán) 1979, medidas provisionales, y 1980, decisión de fondo ${ }^{25}$}

Esta controversia llevada ante la Corte Internacional de Justicia incorpora algunas de las características y los conceptos que hemos desarrollado hasta este punto, pues reúne en sí misma la particular distinción y determinación de lo que puede llegar a ser considerado como un tratado, la forma de atribución de responsabilidad internacional a un Estado y la violación - en los hechos - de obligaciones internacionales expresamente asumidas.

El 4 noviembre de 1979, durante una marcha de protesta frente a la embajada de Estados Unidos en Teherán (Irán), las instalaciones de esta fueron invadidas y tomadas por cientos de manifestantes - en su mayoría estudiantes - armados. Alrededor de cincuenta ciudadanos estadounidenses, una gran parte del cuerpo diplomático y consular, fueron hechos rehenes por un grupo extremista islámico ${ }^{26}$ como muestra de rechazo a lo que llamaban "más de 25 años de continua interferencia de los Estados Unidos en los asuntos internos de Irán, la explotación [...] de nuestro país y numerosos crímenes perpetrados en contra del pueblo iraní..."27, y en clara muestra de apoyo a la revolución liderada por el ayatola Jomeini.

$25 \mathrm{Al}$ tratar este caso y desarrollarlo, usaremos como primera fuente la sentencia United States Diplomatic and Consular Staff in Theran, Judgment, I.C.J. Reports 1980. Haremos referencias textuales o a pie de página a sus correspondientes párrafos y enriqueceremos nuestro análisis con bibliografía complementaria.

26 "The invading group (who subsequently described themselves as 'Muslim Student Followers of the Imam's Policy' [...]" [énfasis nuestro]. (United States Diplomatic and Consular Staff in Theran, Judgment, I.C.J. Reports 1980, para. 17.)

27 Traducción libre de la carta enviada a la Corte Internacional de Justicia por el Gobierno iraní, a través de su Ministerio de Asuntos Exteriores, fechada el 16 de marzo de 1980: "[...] More than 25 years of continual interference by the United States in the internal affairs of Iran, the shameless exploitation of our country, and numerous crimes perpetrated against the Iranian people [...] the deep-rootedness and the essential character of the Islamic Revolution of Iran, a revolution of a whole oppressed nation against its oppressors and their masters, the examination of whose numerous repercussions is essentially and directly a matter within the national sovereignty of Iran". Texto tomado de Weston \& Noyes (2011, p. 326). 
Durante el ataque, y la posterior toma de la embajada, no hubo intento alguno de las fuerzas armadas o policiales del Estado iraní para impedir o tratar de repeler el ataque a las instalaciones. Se afirma que, en los hechos, el personal de seguridad asignado por el Gobierno a la embajada desapareció de la escena ante la multitud y dejó a su suerte a los ocupantes, tanto civiles como diplomáticos ${ }^{28}$.

La pretensión estadounidense ante la Corte, en favor de la inmediata liberación de sus ciudadanos, personal diplomático y consular, estaba sustentada en la supuesta ${ }^{29}$ violación de las obligaciones internacionales por parte de Irán, asumidas por las partes en las Convenciones de Viena de 1961 (Relaciones diplomáticas) y de 1963 (Relaciones consulares).

Invocando el artículo I de los Protocolos Opcionales a las dos convenciones, que prescriben la jurisdicción contenciosa obligatoria de la Corte Internacional de Justicia, se buscaba "traducir una disputa entre Irán y los Estados Unidos específicamente, a una entre Irán y la comunidad internacional en general" ${ }^{30}$, considerando que Estados Unidos e Irán, en el momento de los hechos, eran partes de derecho de las convenciones y no habían registrado ninguna reserva a estas ${ }^{31}$.

El análisis seguido por el cuerpo colegiado fue dividido en dos "fases" que, según sus propias palabras, "sería conveniente examinarlas separadamente": la primera cubre el periodo entre el ataque armado a la embajada, la toma de rehenes, la apropiación de bienes e instalaciones y archivos, hasta la conducta de las autoridades iraníes durante esos hechos; la segunda examinaba los hechos posteriores a la toma de la embajada y de la toma, también, de los consulados estadounidenses en Tabriz y Shiraz.

Respecto a la primera fase del análisis, no se puede afirmar que los militantes, estudiantes y manifestantes armados que atacaron la embajada y luego la tomaron fueran agentes del Gobierno o tuvieran algún cargo oficial que los vinculara a él, por lo que su conducta "lesiva" de los

28 United States Diplomatic and Consular Staff in Theran... para. 17 y 18.

29 Ese era el fundamento jurídico de la pretensión y estaba, como es lógicamente jurídico, sujeta a confirmación por la corte.

30 Weston \& Noyes (2011, p. 341): "It could be that 'the United States agreed to use the Court's decision as a means of translating a dispute between Iran and the United States specifically into one between Iran and the international community generally".

31 United States Diplomatic and Consular Staff in Theran... para. 45. 
derechos estadounidenses de inmunidad y protección diplomática/consular no puede atribuírsele al Estado iraní (bajo esos supuestos) ${ }^{32}$.

Pero esa distinción, hasta este punto, no lo libera de alguna responsabilidad surgida a partir de los ataques, ya que su conducta estaba en conflicto con sus obligaciones internacionales, contraídas en el marco de las Convenciones de Viena de 1961 y 1963, pues fallaron, categóricamente, en brindar protección y ayuda oportuna a la embajada, los consulados, el personal y los bienes de Estados Unidos durante los ataques. Debido a ello, la Corte concluyó que esa falla en tomar las medidas adecuadas de seguridad fue más que una mera negligencia o falta de medios apropiados para tomarlas ("that the failure of the Iranian Government to take such steps was due to more than mere negligence or lack of appropriate means") ${ }^{33}$. Esta omisión de acción estatal - acción que, ejercida en el momento oportuno, hubiera servido para impedir el ataque o repelerlo-, con las posteriores consecuencias que causó, constituyó una clara y seria violación de las obligaciones de Irán para con Estados Unidos prescritas en los artículos 22, párrafo segundo ${ }^{34}$, y 24 al 27 de la Convención de Viena sobre Relaciones Diplomáticas de 1961, y en los artículos 5 y 36 de la Convención de Viena sobre Relaciones Consulares; con lo que se configuró un caso de responsabilidad internacional por incumplimiento de obligaciones.

En la segunda fase del análisis propuesto por la Corte se examina la inacción del Gobierno iraní luego de la toma de la embajada, los consulados y los rehenes. A su juicio, las obligaciones de aquel, conforme al derecho internacional general y las Convenciones de Viena, eran manifiestas, claras. No cumplió con la inmediata obligación (¿también, tal vez, deber?) de realizar todos los esfuerzos que estaban a su alcance y tomar las medidas necesarias para poner fin a la flagrante ruptura del principio de inviolabilidad de las instalaciones y de la inmunidad del personal

32 United States Diplomatic and Consular Staff in Theran... para. 58: "The information before the Court does not, however, suffice to establish with the requisite certainty the existence at that time of such a link between the militants and any competent organ of the State".

33 United States Diplomatic and Consular Staff in Theran... para. 61, 63 y 67.

34 Uno de los más importantes es el artículo 22, numeral segundo, de la Convención de Viena sobre Relaciones Diplomáticas (1961): “2. El Estado receptor tiene la obligación especial de adoptar todas las medidas adecuadas para proteger los locales de la misión contra toda intrusión o daño y evitar que se turbe la tranquilidad de la misión o se atente contra su dignidad". 
diplomático y consular dentro de ellas; esto es, restablecer el orden, regresar las cosas al estado anterior al ataque (restablecer el statu quo de derecho) y ofrecer una reparación por los daños causados ${ }^{35}$. Es evidente que Irán no actuó de ninguna manera conducente a cumplir con cualquiera de sus más elementales obligaciones en el caso que tomamos como ejemplo.

La situación jurídica de Irán fue deteriorándose más todavía, pues funcionarios como el ministro de Asuntos Exteriores ("the action of the students 'enjoys the endorsement and support of the government, because America herself is responsable for this incident"') o el ayatola Jomeini declararon, en los medios de prensa, en favor de las consecuencias ocurridas a causa de las acciones de los militantes en la toma de la embajada y de los rehenes. El 17 de noviembre de 1979, el ayatola Jomeini emitió un decreto que comenzaba con la afirmación de que la embajada estadounidense era un centro de espionaje y conspiración y que las personas que complotan contra su movimiento islámico no gozaban de respeto diplomático internacional, entre otras aseveraciones $^{36}$. Esto no hizo más que configurar, de forma evidente, el vínculo necesario para relacionar a los autores del ataque con el Estado iraní y deducir con esto su responsabilidad bajo el derecho internacional ${ }^{37}$.

35 United States Diplomatic and Consular Staff in Theran... para. 69: “It's plain duty was at once to make every effort, and to take every appropriate step, to bring these flagrant infringements of the inviolability of the premises, archives and diplomatic and consular staff of the United States Embassy to a speedy end, to restore the Consulates at Tabriz and Shiraz to United States Control, and in general to re-establish the status quo and to offer reparation for the damage".

36 United States Diplomatic and Consular Staff in Theran... para. 70 y 73: "73. The seal of official government approval was finally set on this situation by a decree issued on 17 November 1979 by the Ayatollah Khomeini. His decree began with the assertion that the American Embassy was 'a centre of espionage and conspiracy' and that 'those people who hatched plots against our Islamic movement in that place do not enjoy international diplomatic respect'. He went on expressly to declare that the premises of the Embassy and the hostages would remain as they were until the United States had handed over the former Shah for trial and returned his property to Iran".

37 Crawford (2012, p. 555): "Responsibility accrues, quite apart from the operation of other factors, if a state accepts or otherwise adopts the conduct of private persons or entities as its own. The International Court applied this principle to the actions of the militants in Theran Hostages. It is expressed in ARSIWA Article 11, which specifies that the state only becomes responsi- 
The policy thus announced by the Ayatollah Khomeini, of maintaining the occupation of the Embassy and the detention of its inmates as hostages for the purpose of exerting pressure on the United States Government was complied with by other Iranian authorities and endorsed by them repeatedly in statements made in various contexts. The result of that policy was fundamentally to transform the legal nature of the situation created by the occupation of the Embassy and the detention of its diplomatic and consular staff as hostages. The approval given to these facts by the Ayatollah Khomeini and other organs of the Iranian State, and the decision to perpetuate them, translated continuing occupation of the Embassy and detention of the hostages into acts of the State. The militants, authors of the invasion and jailers of the hostages, had now become agents of the Iranian State for whose acts the State itself was internationally responsible. On 6 May 1980, the Minister for Foreign Affairs, Mr. Ghotbzadeh, is reported to have said in a television interview that the occupation of the United States Embassy had been 'done by our nation'. Moreover, in the prevailing circumstances the situation of the hostages was aggravated by the fact that their detention by the militants did not even offer the normal guarantees which might have been afforded by police and security forces subject to the discipline and the control of official superiors $^{38}$ [énfasis nuestro].

Estos actos configuran el supuesto del artículo 11 del Proyecto de artículos sobre responsabilidad internacional de los Estados por hechos internacionalmente ilícitos, de 2001, que en ese entonces era considerado costumbre internacional. Así, una vez determinada la responsabilidad del Estado iraní por el continuo incumplimiento de sus obligaciones internacionales, la Corte, en el párrafo 90 de la sentencia, establece la obligación de reparar por el ilícito en el que ha incurrido y toma esto en consideración en las conclusiones que constituyen su decisión final. La decisión de la sentencia, en su párrafo 95,

ble 'if and to the extent that the State acknowledges and adopts the conduct in question as its own'" Aust (2007, p. 382): "In the Theran Hostages case, the International Court of Justice found that the seizure of the US embassy and the detention of its staff by militant students was attributable to Iran. This was because, by subsequently endorsing the actions and perpetuating them, organs of the Iranian State had made those actions into acts of the State".

United States Diplomatic and Consular Staff in Theran... para. 74. 
prescribe que Irán ha violado, y continúa haciéndolo, varias obligaciones internacionales para con Estados Unidos, contenidas en distintas convenciones internacionales en vigor entre los dos países, así como distintas normas de derecho internacional general. Añade que la violación de estas no solo son atribuibles al Estado iraní, sino también son la base de su responsabilidad para con el Estado demandante y fuente de la obligación de tomar todas las medidas necesarias y conducentes a reparar los hechos ocurridos a partir del 4 de noviembre de 1979 y las consecuencias que les siguieron.

La sentencia ordena también la inmediata liberación de los rehenes, pues fueron hechos prisioneros de manera ilegal, y que se les provea de los medios necesarios para dejar territorio iraní. Finalmente, concluye que el Gobierno de la República Islámica de Irán se encuentra obligado a indemnizar al Gobierno de Estados Unidos por los daños causados y que la forma y los montos de la reparación serán determinados por la Corte, a menos que las partes lleguen a un acuerdo previo.

En el ínterin del proceso y las negociaciones, una de las primeras medidas que tomó el Gobierno del presidente James E. Carter Jr., en un intento de forzar a su contraparte a que liberara a los rehenes, fue congelar bienes y cuentas bancarias iraníes o de sus nacionales que se encontraran en Estados Unidos. Compañías que tenían bienes o grandes cantidades de dinero invertidas en Irán lograron obtener órdenes judiciales que, vinculadas y dirigidas contra los bienes congelados, aseguraban sus pretensiones de indemnización.

Terminada la crisis de los rehenes, tras más de un año de cautiverio, negociaciones y un intento fallido de rescate, con la sentencia y decisiones de la Corte Internacional de Justicia, en un documento jurídico, las partes convinieron en establecer un tribunal -Iran-U.S. Claims Tribunal- que tendría por fin resolver, llevar a término, conocer de las controversias y atender las pretensiones indemnizatorias que surgieran a partir de las consecuencias diplomáticas y pérdidas económicas/comerciales originadas por la crisis de los rehenes.

El tribunal tuvo su carta constitutiva en los llamados "Algiers Accords", de 1981, que consistían en una declaración general hecha por el Gobierno de Algeria - que sirvió como mediador - y contenía los compromisos acordados por Estados Unidos e Irán, así como las obligaciones que entre ellos contraían. 
En su conjunto, la Declaración General y los distintos compromisos constituían un tratado ${ }^{39}$. Estos acuerdos establecían los procedimientos para la liberación de los bienes iraníes congelados, el desistimiento de las órdenes judiciales vinculadas a dichos bienes y la creación de una cuenta/fondo obtenida de los bienes congelados - que serviría al tribunal para pagar las demandas legítimas de ciudadanos estadounidenses en contra de Irán. Las pretensiones y demandas del Gobierno iraní se basan, hasta el día de hoy, principalmente, en las pérdidas sufridas por el abandono del programa de ventas militares de Estados Unidos, que cesó con la revolución de 197940.

The so-called Algiers Accords 1981 constituted treaty arrangements for dealing with claims between Iran and the United States, including the Iran-US Claims Tribunal, were established by: (1) two Declarations by Algeria -a General Declaration and a Declaration concerning the Settlement of Claims- setting out the formal commitments which had been made to it (as a go-between) by Iran and the United States, and Undertakings by the two states, which together constituted a treaty; and (2) an Escrow Agreement between the United States, the Federal Reserve Bank of New York, Bank Markazi, Iran and the Central Bank of Algeria, acting as escrow agent (Aust, 2007, p. 22).

\section{Algunas conclusiones: ¿qué entender, entonces, por "tratado"?}

La primera conclusión que debe venir a la mente del intérprete o del operador jurídico es que en materia de tratados no existe una denomi-

39 Aust (2007, pp. 52 y 410): “The classic form of a single instrument treaty has for long time been joined by treaties drawn in less forAmal ways, such as ex changes of (diplomatic) notes (or letters), which usually consist of one initiating note and one reply note. They can be on matters of national importance or the mundane. Although they are often self-standing, they can be supplementary to another treaty. In fact a treaty can consist of several instruments [...] The Tribunal was established by the so-called Algiers Accords 1981, consisting of a General Declaration by Algeria setting out the commitments made to it by Iran and the United States and Undertakings by the two States, which altogether constituted a Treaty" [énfasis nuestro].

40 Damrosch et al. (2009, p. 549): “The Tribunal is still functioning as of 2009, with the remaining cases principally concerning Iran's extensive claims against the U.S. government for losses incurred when the U.S. military program to Iran was shut down in 1979". 
nación única que determine su contenido y naturaleza. Es decir, un tratado, para efectos del derecho internacional, puede ser llamado "convención", "memorándum de entendimiento", "acuerdo", "cronograma de trabajo", entre otros títulos o encabezados. El trabajo de análisis e interpretación del jurista debe hacerse a la luz del derecho de los tratados (Convención de Viena, artículo 2, 1.a) y de la conjunción de características que desarrollamos en el punto 5.

Uno de los elementos o “características" más importantes que deben tenerse en cuenta es la intención que los Estados puedan tener de crear obligaciones jurídicas gobernadas por el derecho internacional, independientemente de que se encuentren contenidas en uno o más instrumentos/documentos: un intercambio de cartas (bilateral o múltiple), un acuerdo entre dos Estados, acompañado de distintas notas diplomáticas, entre otras combinaciones de instrumentos con relevancia internacional.

Lo importante, repetimos, es la intención jurídica impresa en esos documentos, la que debe ser analizada dentro del contexto y en las circunstancias en las que se forma.

Para efectos de derecho interno, es común encontrar distinciones, "tipos", de tratados explicados o contenidos en normas de rango legal o en la misma Constitución Política de un Estado. Esta realidad es indiferente para el derecho internacional, pues el incumplimiento de una obligación asumida por un Estado es causa suficiente para la configuración de responsabilidad internacional, sin que quepa la posibilidad de alegar normas de derecho interno - sin importar su rango- como origen del incumplimiento.

La Convención de Viena no reconoce dichas distinciones. A efectos de derecho internacional y a la luz de la misma Convención, todos esos documentos, acuerdos o compromisos que hayan sido celebrados por escrito entre Estados son reconocidos como tratados - siempre y cuando concurran las características del primer numeral del artículo 2-, y su incumplimiento tiene como consecuencia la atribución de responsabilidad internacional al Estado infractor.

¿Por qué entonces se diferencia entre las normas internacionales para con el derecho interno, en términos de jerarquía jurídica, si para el derecho internacional tienen una sola categoría, la de obligación internacional? La respuesta a esa pregunta la dejamos pendiente para una futura investigación. 


\section{Referencias}

\section{Doctrina}

Aust, A. (2007). Modern treaty law and practice. (2 ${ }^{\text {nd }}$ edition). Cambridge University Press.

Aust, A. (2010). Handbook of international law. (2nd edition). Cambridge University Press.

Bederman, D. J. (2001). International law in antiquity. Cambridge University Press.

Brownlie, I. (2008). Principles of public international law. (7th edition). Oxford University Press.

Clapham, A. (2012). Brierly's law of nations. (7th edition). Oxford University Press.

Crawford, J. (2012). Brownlie's principles of public international Law. $\left(8^{\text {th }}\right.$ edition). Oxford University Press.

Damrosch, L., Henkin, L., Murphy, S. D., \& Smit, H. (2009). International law cases and materials. (5 $5^{\text {th }}$ edition). West Publishing.

Diez de Velasco, M. (2007). Instituciones de derecho internacional público. (16. ${ }^{\mathrm{a}}$ ed.). Tecnos.

Lowe, V. (2007). International law. Clarendon Series, Oxford University Press.

Novak Talavera, F., \& García-Corrochano Moyano, L. (2003). Derecho internacional público. Lima: Pontificia Universidad Católica del Perú, Fondo Editorial/Instituto de Estudios Internacionales.

Remiro Brotons, A. (2007). Derecho internacional. Tirant lo Blanch.

Reuter, P. (1999). Introducción al derecho de los tratados. Fondo de Cultura Económica.

Truyol y Serra, A. (2008). La sociedad internacional. (Reimp. 2. ${ }^{\text {a }}$ ed.). Alianza Editorial.

Weston Janis, M., \& Noyes J. E. (2011). International law - Cases and commentary. (4 ${ }^{\text {th }}$ edition). West Publishing.

\section{Tratados y proyectos internacionales}

Carta de las Naciones Unidas, 1945.

Convención de Viena sobre Relaciones Diplomáticas, 1961.

Convención de Viena sobre Relaciones Consulares, 1963. 
Convención de Viena sobre Derecho de los Tratados, 1969.

Proyecto de Artículos sobre Responsabilidad Internacional de los Estados por Hechos Internacionalmente Ilícitos, 2001.

\section{Jurisprudencia}

Aegean Sea Continental Shelf, Judgment, I.C.J. Reports 1978.

Maritime Delimitation and Territorial Questions between Qatar and Bahrain, Jurisdiction and Admissibility, Judgment, I.C.J. Reports 1994.

United States Diplomatic and Consular Staff in Tehran, Judgment, I.C.J. Reports 1980. 
